\title{
Combined throat/nasal swab sampling for SARS-CoV-2 is equivalent to nasopharyngeal sampling
}

\author{
A. L. M. Vlek ${ }^{1} \cdot$ T. S. Wesselius ${ }^{2} \cdot$ R. Achterberg ${ }^{3} \cdot$ S. F. T. Thijsen ${ }^{1}$
}

Received: 12 May 2020 / Accepted: 28 June 2020 / Published online: 14 July 2020

(C) Springer-Verlag GmbH Germany, part of Springer Nature 2020

\begin{abstract}
Purpose PCR on a nasopharyngeal sample is the reference method for the detection of SARS-nCoV-2. However, combined throat/nasal sampling as a testing method has several advantages. We compared the combined throat/nasal sampling with nasopharyngeal sampling for detection of SARS-CoV-2 in healthcare workers suspected of COVID-19.

Methods In 107 healthcare workers with symptoms of COVID-19, combined throat/nasal sampling and nasopharyngeal sampling was performed. Detection of SARS-CoV-2 was performed by RT-PCR targeting.

Results A total of 80 healthcare workers $(74.8 \%)$ tested negative with both sampling methods, and 25 healthcare workers (23.4\%) tested positive with both sampling methods. There were two discrepant results with positive PCR in combined throat/ nasal swabs and negative PCR in nasopharyngeal swabs (1.9\%). The $\kappa$ index for concordance between the 2 sampling methods was high (0.95). The median cycle threshold $(\mathrm{Ct})$ value of PCR on nasopharyngeal samples was significantly lower than the $\mathrm{Ct}$ value of PCR on combined throat/nasal samples (19 (IQR 17-20) versus 21 (IQR 18-29) cycles, $p$ value 0.01).

Conclusion Combined throat/nasal swabs yield a similar sensitivity to detect SARS-CoV-2 as nasopharyngeal swabs and are a good alternative sampling method, despite a lower $\mathrm{Ct}$ value for the nasopharyngeal samples.
\end{abstract}

Keywords SARS-CoV-2 $\cdot$ Sampling methods $\cdot$ COVID $\cdot$ Detection

\section{Introduction}

The WHO recommends nasopharyngeal swab sampling as a reference method to detect SARS-CoV-2 [1]. However, combined throat/nasal sampling as an alternative has several advantages. This sampling method is easier to execute, is less invasive, can be performed using universal nylon flock swabs which are routinely used in many hospitals, and can provide an alternative in times of nasopharyngeal swab shortage.

A previous comparison between nasopharyngeal and oropharyngeal swab sampling for SARS-CoV-2 in 353 patients showed that the positive rate of nasopharyngeal swabs was

A. L. M. Vlek

avlek@diakhuis.nl

1 Department of Microbiology and Immunology, Diakonessenhuis Utrecht, Utrecht, the Netherlands

2 Department of Surgery, Diakonessenhuis Utrecht, Utrecht, the Netherlands

3 Short stay unit, Diakonessenhuis Utrecht, Utrecht, the Netherlands higher than that of oropharyngeal swabs (19.0\% vs 6.3\%) [2]. Additionally, higher viral loads have been detected in nasal samples compared with throat samples in an analysis of 17 cases [3] and in a recent study of 146 paired naso- and oropharyngeal samples [4]. However, a study with 94 patients (37\% positive, $n=35$ ) showed identical results for nasopharyngeal and middle nasal turbinate sampling, with cycle threshold $(\mathrm{Ct})$ values that did not differ significantly between the two sampling methods [5]. Also a detailed virological analysis of 9 patients showed no differences in viral loads between naso- and oropharyngeal swabs [6].

To our knowledge, comparing combined throat/nasal sampling with nasopharyngeal sampling for detection of SARSCoV-2 has not been described before.

\section{Methods}

Between 21 and 29 April 2020, combined throat/nasal sampling and nasopharyngeal sampling was performed in 107 healthcare workers from a general hospital in the Netherlands. All subjects showed symptoms of COVID-19 
Table 1 Results of paired SARS$\mathrm{CoV}-2$ testing

\begin{tabular}{cllll}
\hline & & \multicolumn{2}{l}{ Nasopharyngeal swab PCR } & \\
\cline { 3 - 4 } & & Positive & Negative & Total \\
\hline $\begin{array}{c}\text { Combined throat/nasal } \\
\text { swab PCR }\end{array}$ & Positive & $n=25(23.4 \%)$ & $n=2(1.9 \%)$ & $n=27(25.2 \%)$ \\
& Negative & $n=0(0 \%)$ & $n=80(74.8 \%)$ & $n=80(74.8 \%)$ \\
& Total & $n=25(23.4 \%)$ & $n=82(76.6 \%)$ & $n=107(100 \%)$ \\
\hline
\end{tabular}

such as cough, fever, sore throat, dyspnoea, or loss of smell and taste. Healthcare workers were sampled at least $24 \mathrm{~h}$ after the onset of symptoms by a team of nurses who received detailed instructions and training. All healthcare workers were sampled in the early stage of disease, between 24 and $48 \mathrm{~h}$ after symptom onset.

Combined throat/nasal swabs were taken by swabbing the rear wall of the oropharynx and the lower nasal cavity using the same swab. For combined throat/nasal sampling, regular swabs with flocked nylon fiber tip in $1 \mathrm{ml}$ liquid Amies medium were used (Eswab Collection system, Copan, Italy, catalog No. 490CE). Subsequently, nasopharyngeal swabs were inserted in one nostril until reaching the back of the nasopharyngeal cavity and rotated before removal. For nasopharyngeal sampling, an ultra-thin applicator swab with flocked nylon fiber tip in $1 \mathrm{ml}$ liquid Amies medium was used (Eswab Collection system, Copan, Italy, catalog No. 483C). Swabs were transported to the microbiology laboratory where PCR testing was performed on the same day.

Detection of SARS-CoV-2 was performed by RT-PCR targeting the E gene. RNA was isolated using the Magnapure MP24 total NA kit (Roche, Almere, the Netherlands) according to the manufacturer's instructions. PCR was performed as described by Corman et al. [7]. Realtime PCR was performed using the ABI Prism 7000 Sequence Detection System (Applied Biosystems, Foster City, CA, USA). A Ct value of 40 was used as the cutoff. No creeping signals or indications for contamination were observed in the PCR.

Results for $\mathrm{Ct}$ values are expressed as median with interquartile range (IQR). Summary data were calculated using a Wilcoxon signed ranks test. Statistical analyses were performed with SPSS version 23.0 (Windows, Chicago, USA).

\section{Results}

The median age of the 107 subjects was 34 years (range 19-63 years). A total of 80 healthcare workers $(74.8 \%)$ tested negative with both sampling methods, and 25 healthcare workers $(23.4 \%)$ tested positive with both sampling methods. There were two discrepant results with positive PCR in combined throat/nasal swabs and negative PCR in nasopharyngeal swabs (1.9\%) (Table 1). The $\kappa$ index for concordance between the 2 sampling methods was high (0.95).

Median Ct value of PCR on nasopharyngeal samples was 19 (range 14-31; IQR 17-20), compared with 21 (range 15-37; IQR 18-29) for combined throat/nasal samples (Fig. 1). The median cycle threshold $(\mathrm{Ct})$ value of PCR on nasopharyngeal samples was significantly lower than the $\mathrm{Ct}$ value of PCR on combined throat/nasal samples ( $p$ value 0.01 ). The combined throat/nasal swabs that were positive with negative nasopharyngeal swab PCR had $\mathrm{Ct}$ values of 32 and 37.

A limitation to our study is the limited number of patients as well as positive samples. However, as we did not observe any positive nasopharyngeal samples with negative throat/nasal swab, double sampling of additional healthcare workers was judged unnecessary. In this study, two different sampling methods were compared in order to determine less invasive and easier to perform sampling techniques. In future studies assessing the utility of different patient samples for the diagnosis of COVID-19, it would be interesting to compare the combined throat/ nasal swab with saliva specimens as well.

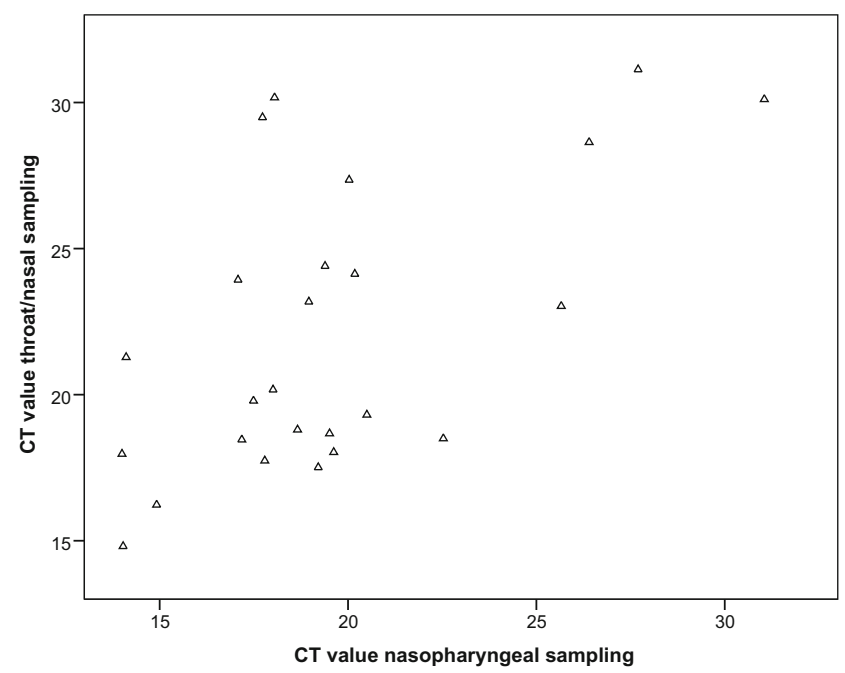

Fig. $1 \mathrm{Ct}$ values of paired samples with positive SARS-CoV-2 in both samples 


\section{Conclusion}

Our study shows that combined throat/nasal swabs yield a similar sensitivity to detect SARS-CoV-2 as nasopharyngeal swabs, despite a lower $\mathrm{Ct}$ value for the nasopharyngeal samples.

Data availability Yes.

\section{Compliance with ethical standards}

Conflicts of interest The authors declare that they have no conflict of interest.

Code availability NA.

\section{References}

1. World Health Organization. Laboratory testing for 2019 novel coronavirus (2019-nCoV) in suspected human cases. Interim guidance. 19 March 2020. Available at: https://www.who.int/publicationsdetail/laboratory-testing-for-2019-novel-coronavirus-in-suspectedhuman-cases-20200117 [Accessed 1 May 2020]

2. Wang X, Tan L, Wang X, Liu W, Lu Y, Cheng L, Sun Z (2020) Comparison of nasopharyngeal and oropharyngeal swabs for SARSCoV-2 detection in 353 patients received tests with both specimens simultaneously. Int J Infect Dis 94:107-109
3. Zou L, Ruan F, Huang M, Liang L, Huang H, Hong Z, Yu J, Kang M, Song Y, Xia J, Guo Q, Song T, He J, Yen HL, Peiris M, Wu J (2020) SARS-CoV-2 viral load in upper respiratory specimens of infected patients. N Engl J Med 382:1177-1179

4. Patel M, Carroll D, Ussery E, Whitham H, Elkins C, Noble-Wang J, Rasheed J, Lu X, Lindstrom S, Bowen V, Waller J, Armstrong G, Gerber S, Brooks J (2020) Performance of oropharyngeal swab testing compared to nasopharyngeal swab testing for diagnosis of COVID-19. Clin Inf Dis [online ahead of print]

5. Vermeiren C, Marchand-Senécal X, Sheldrake E, Bulir D, Smieja M, Chong S, Forbes JD, Katz K (2020) Comparison of Copan Eswab and FLOQswab for COVID-19 PCR diagnosis: working around a supply shortage. J Clin Microbiology 58(6):e00669-20. https://doi. org/10.1128/JCM.00669-20

6. Wolfel R, Corman V, Guggemos W, Seilmaier M, Zange S, Muller M, Niemeyer D, Jones T, Vollmar P, Rothe C, Hoelscher M, Bleicker T, Brunink S, Schneider J, Ehmann R, Zwirglmaier K, Drosten C, Wendtner C (2020) Virological assessment of hospitalized patients with COVID-19. Nature 581:465-469

7. Corman VM, Landt O, Kaiser M, Molenkamp R, Meijer A, Chu DK, Bleicker T, Brünink S, Schneider J, Schmidt ML, Mulders DG, Haagmans BL, van der Veer B, van den Brink S, Wijsman L, Goderski G, Romette JL, Ellis J, Zambon M, Peiris M, Goossens H, Reusken C, Koopmans MP, Drosten C (2020) Detection of 2019 novel coronavirus (2019-nCoV) by real-time RT-PCR. Euro Surveill $25: 2000045$

Publisher's note Springer Nature remains neutral with regard to jurisdictional claims in published maps and institutional affiliations. 